\title{
Éditorial
}

\section{Répondez à l'appel de Vancouver !}

\author{
Jacky Samson*
}

"On fait la science avec des faits, comme on fait une maison avec des pierres, mais une accumulation de faits n'est pas plus une science qu'un tas de pierres n'est une maison. ॥

Henri Poincaré

Le 15 juin 2013, la revue Médecine Buccale Chirurgie Buccale a ouvert le site de soumission en ligne et, dorénavant, tous les articles adressés à la revue doivent être soumis par cette voie. Toute la procédure suivie par le manuscrit (soumission à 2 relecteurs, commentaires des relecteurs, retour aux auteurs du manuscrit avec la décision du Comité de rédaction, relecture des épreuves) se fait par voie électronique comme dans toutes les revues internationales référencées. Pour respecter les recommandations du groupe de Vancouver (International Committee of Medical Journal Editors ou ICMJE), les auteurs trouveront sur le site de la revue dans la colonne située sur la gauche différentes rubriques (considérations éthiques, conseils aux auteurs, transfert des droits d'auteurs) avec, pour chaque rubrique, une version française et une version anglaise. Malheureusement, pour le site de soumission, il n'y a qu'une version anglaise. En effet, il n'existe que deux programmes de soumission en ligne et ils sont tous les deux en anglais. En raison des systèmes d'alerte (pour le Comité de rédaction, pour le dialogue avec les relecteurs et pour les échanges avec les auteurs) qui sont couplés au formulaire de soumission, il n'est pas possible d'envisager une traduction en français sans devoir reconstruire tout le programme. Pour la déclaration de conflits d'intérêts, nous avons adopté celle du ICMJE et, un peu pour les mêmes raisons, nous avons conservé la version originale. La HAS a mis au point un formulaire de « déclaration publique d'intérêts » qu'elle soumet aux « membres des commissions spécialisées et du comité de validation des recommandations de bonne pratique, aux experts et à toute personne qui collabore ... » mais il est encore plus complexe, et donc plus long (11 pages), que celui de l'ICMJE et, de plus, il n'existe pas de version électronique.

Au début, tout ceci vous paraitra bien lourd mais, avec le temps, la procédure vous semblera de plus en plus simple. Certes, in fine, elle sera toujours plus lourde, et donc plus chronophage, que la procédure que nous avons utilisé jusqu'à maintenant. Cette dernière avait été allégée au maximum et ne respectait guère plus les conceptions ou recommandations, scientifiques et juridiques, actuelles. Tout ceci constituait le premier pas vers le référencement. Le second dépend surtout de vous : pour le référencement, il faut respecter la forme et veillé aussi sur le fond.

Commençons par la partie où la forme et le fond sont étroitement imbriqués. Abordons d'abord la qualité d'auteur : trop souvent le nombre d'auteurs est disproportionné par rapport à l'importance du sujet traité et bien des auteurs n'ont pas apporté une contribution suffisante pour mériter ce titre. La qualité d'auteur invité (" auteur cadeau » ou « guest author » [1]) et, à l'opposé, le « ghost writing » (surtout pratiqué par les firmes pharmaceutiques [2]) constituent des infractions à l'intégrité scientifiques [3]. De plus en plus de revues demandent des précisions sur la contribution de chacun des auteurs dans le travail soumis pour publication. Et pour justifier leur qualité, les auteurs doivent remplir les trois conditions suivantes : «1. (avoir apporté) une contribution substantielle à la conception, à la méthode et à l'acquisition des données ou à leur analyse et leur interprétation ; 2 . (avoir réalisé) la rédaction de l'article ou sa révision critique impliquant une contribution importante au contenu intellectuel ; et 3. (avoir donné) son approbation à la version finale. » [4]. « Tous les auteurs qui ne remplissent pas les trois critères de paternité doivent être cités dans la section remerciements. » [4]. Toujours pour la forme, il faut s'efforcer de présenter les articles selon la structure IMRAD (Introduction, Methods, Resultats, And, Discussion) établie initialement pour les articles originaux. Le respect de cette structure, qui traduit la logique scientifique, oblige à exposer clairement le travail réalisé, que ce soit pour un travail de recherche ou pour une revue systématique [1]. Quant au cas clinique qui rapporte une observation originale,

\footnotetext{
*Correspondance : jacky.samson@unige.ch
} 
ou quelquefois plusieurs, son plan comporte trois parties (introduction, observation, discussion) beaucoup moins développées. Mais ce plan est également proche de la structure IMRAD [1].

Pour le fond, rappelons deux règles de base [4]. La plupart des revues exigent que le manuscrit n'ait pas été soumis simultanément à d'autres revues. De même, il faut éviter les publications redondantes qui reprennent une grande partie de données déjà publiées ; dans sa forme caricaturale, la publication redondante est constituée par la simple traduction d'un article paru dans une autre langue. Il existe une autre démarche qui consiste à publier les résultats d'une étude de façon fragmentée dans plusieurs articles (« salami factor »). Ces deux démarches cherchent à répondre, au mépris de l'intégrité scientifique, au diktat des index bibliométriques que l'on retrouve souvent sous une forme plus percutante « publish or perish ». Après l'obtention du référencement, il y a une autre étape primordiale, c'est l'évaluation de l'impact factor (IF) de notre revue. L'IF n'a jamais été conçu pour évaluer la qualité des articles ou pour favoriser l'évolution d'une carrière mais, comme son fondateur, E Garfield, l'a souvent rappelé, pour évaluer la notoriété des revues sans réellement évaluer les articles. Néanmoins, la notoriété de la plupart des revues dépend assez directement de la qualité des articles publiés. Partant, nous vous invitons à être vigilants sur la qualité des articles que vous allez soumettre afin que notre revue devienne officiellement une des meilleures revues dans notre domaine.
Notre spécialité vient d'atteindre sa majorité. Il faut qu'à son tour, la revue de notre spécialité devienne majeure. Et cela ne peut se faire sans votre participation et votre investissement. Rédiger un article reste toujours une épreuve mais, comme cette épreuve contribue grandement à la formation des auteurs, nous vous invitons à répéter cet exercice sans retenue. Pour vous stimuler, s'il en était besoin, méditez cette pensée de Virgile : «On se lasse de tout, excepté d'apprendre ». Ainsi, l'IF de notre revue deviendra très rapidement honorable.

\section{Références}

1. Maisonneuve $\mathrm{H}$, Lorette $\mathrm{G}$, Maruani A, Huguier M. La rédaction médicale, $5^{\mathrm{e}}$ ed. Doin Editeurs, Rueil-Malmaison, 2010.

2. Pasca E. Ghostwriting (auteurs fantôme) et autres méthodes de manipulation et influence sur la recherche médicale et l'information. pharmacritique.20minutes-blogs.fr/archive/2012/10/10/ghostwriting-auteurs-fantome-et-autres-methodes-de-manipulat.html. 10/ 10/2012.

3. Académie suisse des sciences. Qualité d'auteur des publications scientifiques. Analyse et recommandations. www.academiessuisses.ch/fr/index/Aktuell/News.html. 30 mai 2013.

4. Comité international des rédacteurs de revues médicales. Exigences uniformes pour les manuscrits soumis aux revues biomédicales : rédaction et édition de la publication biomédicale. www.icmje.org/french.pdf. 